\title{
Exploiting the Capture Effect to Improve WLAN Throughput
}

\author{
Paul Patras, Hanghang Qi and David Malone \\ Hamilton Institute \\ National University of Ireland Maynooth, Ireland \\ Email:\{Paul.Patras,Hanghang.Qi,David.Malone\}@nuim.ie
}

\begin{abstract}
In practical WLAN deployments, the capture effect has been shown to enhance the performance of stations residing close to the $A P$, while putting at disadvantage the distant nodes. In this paper, we introduce an analytical model to characterise the performance of $\mathbf{8 0 2 . 1 1}$ devices with heterogeneous capture probabilities and different network loads, and explore the interaction between the MAC operation and PHY capture. Unlike previous studies, we reveal that the throughput of stations experiencing low capture probabilities can also benefit from the capture effect when the stations retaining high capture probabilities are not saturated. Following these findings, we design a power-hopping scheme for 802.11 MAC that exploits the benefits of the capture effect to improve performance in dense deployments where nodes experience similar channel conditions. We investigate the potential gains of this mechanism by implementing a practical approximation using commercial off-the-shelf hardware and open-source drivers and, by conducting experiments in a real testbed, we show that our scheme can significantly outperform the standard 802.11 protocol in terms of throughput.
\end{abstract}

\section{INTRODUCTION}

In the analysis of the IEEE 802.11 MAC, it is often assumed that whenever more than one station transmits at the same time, the transmitted frames fail to be decoded at the receiver due to collision (e.g. [1]-[4]). In practice, at the physical layer, the phenomenon known as capture effect is frequently encountered when frames arrive at destination with different power levels and the strongest signal can be demodulated, despite the presence of other interfering signals. Thus, some packets can be successfully received even when simultaneous transmissions occur, provided the difference in their signal strength is sufficiently large.

While aspects of the capture effect have been widely studied in the past in the context of mobile radio environments, e.g. [5], [6], recent works [7]-[10] propose analytical models of the IEEE 802.11 protocol that seek to predict the performance of WLANs under such conditions, showing that capture effect can reduce the number of failures due to collisions, thereby increasing the overall network throughput. These analytical results are further confirmed by

\footnotetext{
The research leading to these results has received funding from the European Community's $7^{\text {th }}$ Framework Programme (FP7-ICT-2009-5) under grant agreement n. 257263 (FLAVIA project) and the Irish HEA PRTLI Cycle 4 FutureComm.
}

experimental studies that examine the capture phenomenon comprehensively with real deployments [11]-[13].

In this paper, we further explore 802.11's behaviour under the capture effect and, unlike previous works, we show that capture effect can not only improve the overall throughput of the network, but may also enhance the performance of the nodes that deliver frames at a low signal level, when the stations experiencing better link qualities with the receiver are lightly loaded. To this end, we first conduct an analysis of the 802.11 operation, adopting a renewal-reward process [3] to model the binary exponential backoff scheme of the MAC protocol with multiple classes of stations that experience heterogeneous capture probabilities. Based on this analysis, we show that, depending on the network load, the throughput attained by the stations residing further away from the access point (AP) is not always degraded due to nodes located near the AP capturing the channel, but, on the contrary, the capture effect can also reduce the collision rate encountered by the distant users, thereby providing them with larger throughput. By examining the impact of the traffic load on the transmission attempt rate and success probability, we provide valuable insights into the observed behaviour.

Second, we design a simple yet effective power-hopping MAC/PHY scheme that exploits the identified protocol behaviour to boost the WLAN performance in dense deployments, where stations undergo similar channel conditions. Specifically, our proposal preserves the 802.11 MAC rules, but alternates between a low and a high transmission power level when sending frames, thus randomly causing capture at the receiver. We model this enhancement using a Bianchitype Markov chain [1] and show that our scheme lessens the impact of collisions, providing significantly better throughput performance as compared to the standard 802.11 protocol. To investigate the effectiveness of our mechanism, we develop a practical proof-of-concept implementation using open-source drivers and off-the-shelf 802.11 hardware, and assess its potential by conducting experiments in a smallscale testbed. The obtained results show that PH-MAC can achieve noteworthy throughput gains over the default 802.11 scheme in realistic scenarios, while involving no hardware modifications and only modest programming effort.

The rest of the paper is organised as follows. In Section II, we present the network model considered for our analysis, in 
Section III we undertake an analytical and numerical study of the 802.11 performance with heterogeneous capture and, in Section IV we introduce the power-hopping MAC scheme that we implement and validate experimentally with real devices. Finally, Section V provides concluding remarks.

\section{NeTWORK MODEL}

In this section, we provide an overview of the network model and the assumptions used in the performance analysis that we conduct. We consider the case of infrastructure 802.11 wireless networks, i.e. all transmissions are to/from the AP. We start by introducing relevant aspects of the IEEE 802.11 protocol with the DCF (Distributed Coordination Function) operation, which is the default channel access scheme currently employed in WLANs [14] and then explain how capture is accounted for in our system.

\section{A. IEEE 802.11 DCF}

DCF uses a CSMA/CA (Carrier Sense Multiple Access with Collision Avoidance) MAC protocol with binary slotted exponential backoff. Briefly, when a station having packets to send senses the wireless medium idle for a period of DIFS (Distributed Inter-frame Space), it initialises a backoff counter with a random value uniformly distributed in the $[0, C W]$ interval and enters a count down state. Specifically, as long as the medium remains idle, the node decrements its backoff counter by one after each slot time $\sigma$, and suspends the countdown if the medium becomes busy. The countdown is resumed once the medium is idle again and when the backoff counter reaches zero, the station transmits. The $C W$ parameter is called the contention window and its value is set to a default $C W_{\min }$ upon the first transmission attempt.

If a transmitted frame is successfully received, the destination sends back an acknowledgement (ACK) frame after a period of SIFS (Short Inter-frame Space) to notify the sender of the correct reception. In case the backoff counters of two or more stations reach zero simultaneously, the stations transmit in the same time slot and thus a collision occurs. If the destination cannot decode a frame, no ACK is sent back. When a station does not receive an ACK within a predetermined timeout, it will double its $C W$ value and re-enter the backoff process, attempting to resend the frame. Upon consecutive unsuccessful transmission attempts, $C W$ can be doubled up to a maximum value $C W_{\max }=2^{m} C W_{\min }$, where $m$ denotes the maximum backoff stage. If further attempts fail, $C W$ is maintained

Table I 802.11B PHY/MAC PARAMETERS

\begin{tabular}{lr|lr}
\hline \hline$C W_{\min }$ & 31 & Slot time $(\sigma)$ & $20 \mu \mathrm{s}$ \\
\hline$m$ & 5 & Data rate & $11 \mathrm{Mb} / \mathrm{s}$ \\
\hline DIFS & $50 \mu \mathrm{s}$ & PLCP duration & $96 \mu \mathrm{s}$ \\
\hline SIFS & $10 \mu \mathrm{s}$ & ACK duration & $106 \mu \mathrm{s}$ \\
\hline \hline
\end{tabular}

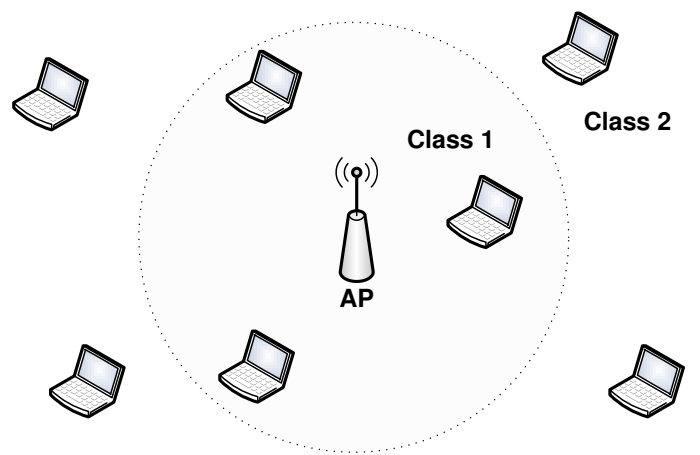

Figure 1. Classes of stations with heterogeneous capture probabilities.

at the maximum value and eventually the frame is discarded after a consecutive retry limit $K$ is exceeded. Otherwise, if the transmission is successful, the $C W$ is reset to $C W_{\min }$. The values of the involved parameters are summarised in Table I for the HR/DSSS (802.11b) physical layer [14].

\section{B. System Assumptions}

We consider a WLAN where nodes are within carrier sense range of one another, thus the RTS/CTS mechanism is not necessary, and assume ideal channel conditions, therefore losses are only caused by collisions. Without loss of generality we assume stations send fixed size packets and employ a single PHY rate. These assumptions are commonly used in the literature [1]-[4] and could be relaxed, but we expect that they do not significantly change our conclusions.

We analyse the 802.11 MAC behaviour with heterogeneous capture, distinguishing two classes of nodes that experience dissimilar capture probabilities, as in the example illustrated in Fig. 1. Note that our analysis can be easily extended to account for multiple groups of stations (fully heterogeneous network) by augmenting [4] with a suitable set of capture rules.

Given their placement with respect to the AP, nodes in Class 1 can capture the channel over nodes in Class 2. Consequently, when concurrent transmissions occur, the following outcomes are possible:

- If two or more stations in Class 1 transmit $\Rightarrow$ collision;

- If a station in Class 1 and respectively a node in Class 2 transmit $\Rightarrow$ station in Class 1 captures with probability $\alpha$; transmission of station in Class 2 fails;

- If a station in Class 2 transmits simultaneously with any other station $\Rightarrow$ transmission fails.

In our analysis, we treat the capture probability $\alpha$ as a model parameter, while methods given in e.g. [8], [9] can be employed for accurate estimation. In what follows, we study the 802.11 throughput performance under different network loads in the presence of capture, to identify those scenarios in which all classes of stations could benefit from this effect. 


\section{Performance Analysis of 802.11 With Heterogeneous CAPTURE}

To study the performance of a WLAN where stations experience dissimilar capture probabilities, we first present a throughput model that accounts for this effect and then employ numerical analysis to illustrate that gains can be achieved in such circumstances, depending on the volume of traffic nodes generate. By investigating the relationship between the success probabilities, transmission attempt rates and arrival processes, we provide valuable insights into the observed behaviour.

\section{A. Throughput Model}

We propose a realistic model of 802.11 operation that, unlike previous works, allows nodes with heterogeneous capture probabilities. In the absence of capture effect, simultaneous frame transmissions fail due to collisions. In this case, the conditional failure (collision) probability $p$ experienced by a transmitted frame is given by [1]:

$$
p=1-(1-\tau)^{n-1},
$$

where $\tau$ is the stationary probability that a station transmits in a randomly chosen slot time and $n$ is the number of stations in the WLAN.

In the presence of the capture effect, a transmission of a station can be successful even when another station transmits simultaneously. However, only the frame received with a higher signal level will be decoded by the AP, while the other transmission will result in failure. We denote by $p_{1}$ and $p_{2}$ the conditional failure probabilities for the two classes of stations considered. The corresponding transmission probabilities of stations in Class 1 and Class 2 are given by $\tau_{1}$ and $\tau_{2}$, respectively, and can be related as follows:

$$
\left\{\begin{aligned}
1-p_{1}= & \left(1-\tau_{1}\right)^{\left(n_{1}-1\right)}\left(1-\tau_{2}\right)^{n_{2}} \\
& +\left(1-\tau_{1}\right)^{\left(n_{1}-1\right)}\left(1-\left(1-\tau_{2}\right)^{n_{2}}\right) \cdot \alpha \\
1-p_{2}= & \left(1-\tau_{1}\right)^{n_{1}}\left(1-\tau_{2}\right)^{\left(n_{2}-1\right)}
\end{aligned}\right.
$$

where $\alpha$ is the probability that a station from Class 1 captures the channel over stations from Class 2 and $n_{j}, j \in\{1,2\}$, denotes the number of stations belonging to Class $j$. We model the protocol behaviour differently from [7], [8] and [10], where the failure probability is computed by subtracting a capture probability from the collision probability, i.e. $p=1-(1-\tau)^{n-1}-p_{\text {cap }}$. Instead, we treat a fraction of the collisions as resulting in capture.

Next, we use a renewal-reward approach to analyse the 802.11 binary exponential backoff scheme. The advantage of using this approach is that it does not require the direct calculation of the stationary distribution of the Markov Chain [3], [15], but instead we calculate the expected number of slots and transmissions between the renewal events, i.e. the completion of packet transmissions. Let $p$ be a station's conditional failure probability. The expected number of attempts to transmit a packet is

$$
E(R)=1+p+p^{2}+\ldots+p^{K},
$$

where $K$ is the maximum number of retry attempts. The expected number of slots used during backoff is

$$
E(X)=t_{i}+b_{0}+p b_{1}+p^{2} b_{2}+\ldots+p^{k} b_{k}+\ldots+p^{K} b_{K},
$$

where $b_{i}$ is the mean length of backoff stage $i$ expressed in slots and $t_{i}$ is the mean idle time that a station waits for a packet after transmission. Thus, we can express the transmission attempt rate of the station as

$$
\tau=\frac{E(R)}{E(X)}=\frac{1+p+p^{2}+\ldots+p^{K}}{t_{i}+b_{0}+p b_{1}+p^{2} b_{2}+\ldots+p^{K} b_{K}} .
$$

We apply the above equation to the considered classes of stations to relate each $\tau_{j}$ to the corresponding $p_{j}$.

Neglecting post-backoff and assuming no buffering, we can write

$$
t_{i}=q\left(1+2(1-q)+3(1-q)^{2}+\ldots\right)=\frac{1}{q},
$$

where $q$ is the probability that a new frame arrives in a uniform slot time $E_{s}$, given by

$$
E_{s}=P_{i} \sigma+P_{s} T_{s}+P_{f} T_{f},
$$

in which $\sigma, T_{s}$ and $T_{f}$ are the average durations of an idle slot, a successful transmission and a failure, respectively, and $P_{i}, P_{s}$ and $P_{f}$ are the corresponding probabilities, given by

$$
\begin{aligned}
P_{i} & =\left(1-\tau_{1}\right)^{n_{1}}\left(1-\tau_{2}\right)^{n_{2}}, \\
P_{s} & =n_{1} \tau_{1}\left(1-p_{1}\right)+n_{2} \tau_{2}\left(1-p_{2}\right), \\
P_{f} & =1-P_{i}-P_{s},
\end{aligned}
$$

$T_{s}$ and $T_{f}$ can be expressed as

$$
\begin{gathered}
T_{s}=T_{P L C P}+\frac{E[L]}{C}+S I F S+T_{A C K}+D I F S \\
T_{f}=T_{P L C P}+\frac{E[L]}{C}+T_{A C K_{-} \text {timeout }},
\end{gathered}
$$

where $T_{P L C P}$ is the duration of the PLCP (Physical Layer Convergence Protocol) preamble and headers, $L$ is the average frame length, $T_{A C K}$ is the duration of an acknowledgment, $C$ is the PHY rate and $T_{A C K_{-} \text {timeout }}$ is a physical layer constant.

Given $C W_{\max }=2^{m} C W_{\min }$ and using $W=C W_{\min }$ to simplify notation, we have $b_{k}=2^{k} W / 2, \forall k \geq 0$ and, assuming infinite backoff $(K \rightarrow \infty)$, we obtain:

$$
\left\{\begin{array}{l}
\tau_{1}=\frac{2\left(1-2 p_{1}\right)}{W\left(1-p_{1}-p_{1}\left(2 p_{1}\right)^{m}\right)+\frac{2\left(1-2 p_{1}\right)\left(1-p_{1}\right)}{q}}, \\
\tau_{2}=\frac{2\left(1-2 p_{2}\right)}{W\left(1-p_{2}-p_{2}\left(2 p_{2}\right)^{m}\right)+\frac{2\left(1-2 p_{2}\right)\left(1-p_{2}\right)}{q}} .
\end{array}\right.
$$




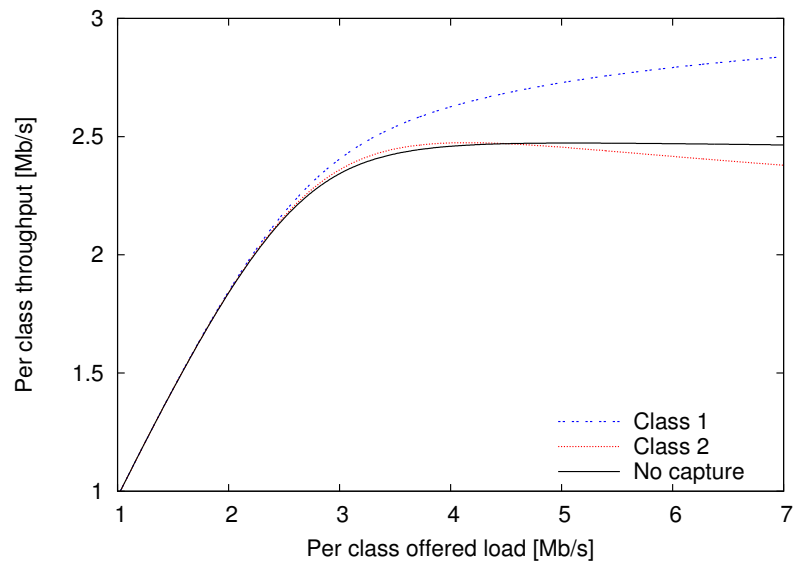

Figure 2. Throughput performance with and without capture effect.

Combining (2) and (11), we can solve $\left(\tau_{1}, p_{1}, \tau_{2}, p_{2}\right)$ and compute the total throughput of the network as follows:

$$
S=\frac{P_{s} E[P]}{E_{s}},
$$

where $E[P]$ is the expected size of the payload. The above completes our throughput model. Next, we study the impact of the offered load on the performance attained by the two classes of stations with capture effect.

\section{B. Numerical Results and Discussion}

Using the proposed model, we consider an example scenario of a network with 10 stations evenly distributed among classes (i.e. $n_{1}=5, n_{2}=5$ ), and analyse numerically the throughput attained by each class as the offered load is varied. ${ }^{1}$ We assume stations employ the $802.11 \mathrm{~b}$ system parameters, as summarised in Table I, and are transmitting packets of fixed size, $E[P]=500$ bytes. We consider identical load at each node and $\alpha=0.75$, i.e. a Class 1 station can capture the channel over Class 2 stations $75 \%$ of the time. ${ }^{2}$ The results are shown in Fig. 2 where we also plot for comparison the throughput performance of an identical network setup but without capture effect (i.e. $\alpha=0$ ). As expected, the throughput of Class 1 is always above the throughput achieved by the same group of stations in a capture free scenario. However, what is more interesting to remark, is that the throughput of Class 2 can also be larger in the presence of capture, depending on the offered load of Class 1 stations. Note that, although the observed differences may appear small, it is worth comprehending what drives these improvements, as we will show later that capture can be exploited when nodes experience similar channel conditions to enhance the overall network performance.

\footnotetext{
${ }^{1}$ We relate the arrival rate per slot $q$ to the actual packet arrival rate per second $\lambda$, with $\lambda=-\log (1-q) / E_{s}$. Assuming all the traffic is independent and Poisson, the offered load is obtained as $\lambda E[P]$.

${ }^{2}$ Simulations conducted with different values of $\alpha$ yield similar results.
}

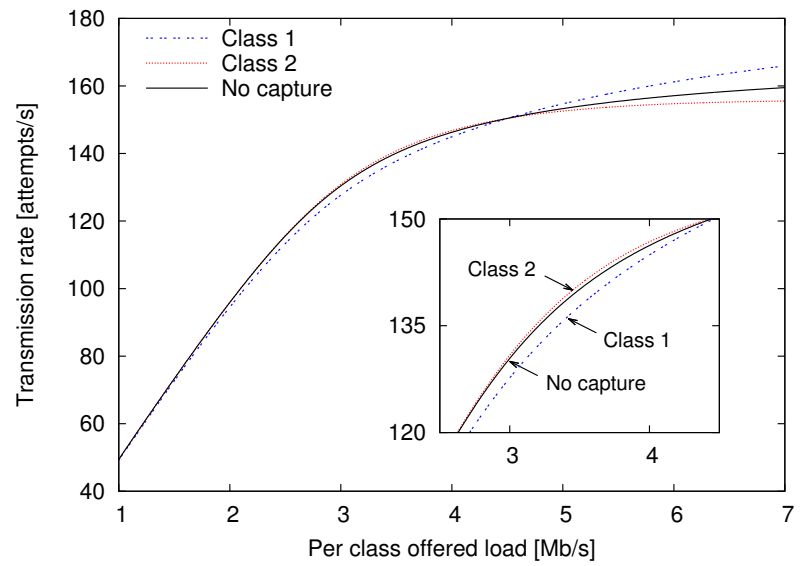

Figure 3. Transmission rate, $\tau / E_{s}$.

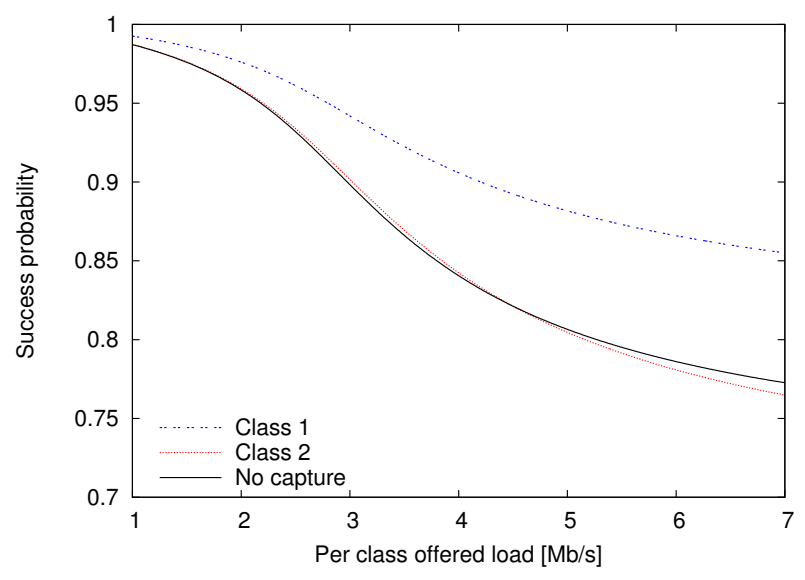

Figure 4. Success probability, $(1-p)$.

To better understand the perceived behaviour, let us first write the throughput for a single node in Class $j$ as:

$$
S_{j}=E[P] \frac{\tau_{j}}{E_{s}}\left(1-p_{j}\right) .
$$

Since $E[P]$ is fixed, the throughput variation can be explained by changes in the transmission rate, $\tau / E_{s}$, and in the success probability, $1-p$. Note that, because of the random access mechanism, these two quantities depend on one another, as already explained in Section III-A. Therefore, to provide further insight into the evolution of the two terms, we separately plot these in Figs. 3 and 4, respectively, for the two classes of stations under study.

For the case of Class 1, Fig. 3 shows that the transmission rate can be smaller in the presence of capture if the network is lightly loaded. At the same time, Fig. 4 illustrates that the success probability seen by Class 1 is always higher than the one experienced by Class 2 in the network with capture. Thus, the overall throughput improvements of Class 1 are a nontrivial combination of tradeoffs between transmission rate and probability of success. 
For the case of Class 2, similar tradeoffs occur. Namely, while the failure probability, $p$, increases with the offered load, the success probability, $1-p$, can actually become higher than in the equivalent network without capture, allowing the possibility of improved throughput over the capture free case. At the same time, the transmission rate of Class 2 is equal or lower in the presence of capture as compared to the capture free case. As the observed transmission rate effectively stops increasing at a low offered load while the failure probability continues to increase, this explains the peak in the throughput of Class 2 seen in Fig. 2.

To conclude this section, we will study the relationship between transmission and conditional failure probabilities. This will offer insight into how the throughput gains are related to differences in failure probability between the two classes and to the network load.

It is worth noting that, at very low offered loads, we observe that the transmission rate of Class 1 is lower than that of Class 2. This may be initially regarded as surprising, since both classes have the same offered load and Class 2 sees a higher failure probability. In particular, as stations backoff upon failed attempts, higher failure probabilities are usually associated with lower transmission probabilities. Given this counter-intuitive behaviour, we further analyse the relationship between transmission and failure probabilities.

If we take the derivative of (5) with respect to $p$, we obtain

$$
\begin{aligned}
\frac{d \tau}{d p} & =\frac{\left(\sum_{k=0}^{K} b_{k} p^{k}+\frac{1}{q}\right)\left(\sum_{l=1}^{K} l p^{l-1}\right)}{\left(\sum_{k=0}^{K} b_{k} p^{k}+\frac{1}{q}\right)^{2}} \\
& -\frac{\left(\sum_{k=0}^{K} p^{k}\right)\left(\sum_{l=1}^{K} l b_{l} p^{l-1}\right)}{\left(\sum_{k=0}^{K} b_{k} p^{k}+\frac{1}{q}\right)^{2}} .
\end{aligned}
$$

Kumar et al. proved that in saturation (i.e. $q=1$ ), if $b_{l}>=b_{k}, \forall l>k$ and $b_{k}$ are not all equal, then $\tau$ is strictly decreasing with $p$ when $p \in(0,1)$ [3]. Therefore, the expression in (14) will be negative.

Conversely, when the network is lightly loaded, from our previous observation about the attempt rates of the two classes we conjecture that $\tau$ must not be decreasing. If we evaluate the numerator of (14) when the derivative changes sign, we can write:

$$
\begin{array}{r}
\left(p^{m}\left(4 m 2^{m} p+(-2 m-2) 2^{m}\right)+2\right) q^{2} W \\
+\left(8 p^{2}-8 p+2\right) q^{2}+\left(-8 p^{2}+8 p-2\right) q=0 .
\end{array}
$$

In a lightly-loaded case (i.e. $p \approx 0$ ), the above is equivalent to:

$$
2 q^{2} W+2 q^{2}-2 q=0,
$$

which has a root at $q_{0}=1 /(W+1)$. Intuitively, this tells us that when the arrival rate is smaller than one packet every $W+1$ slots, then increasing $p$ can increase $\tau$, due to the increased number of retransmission attempts.

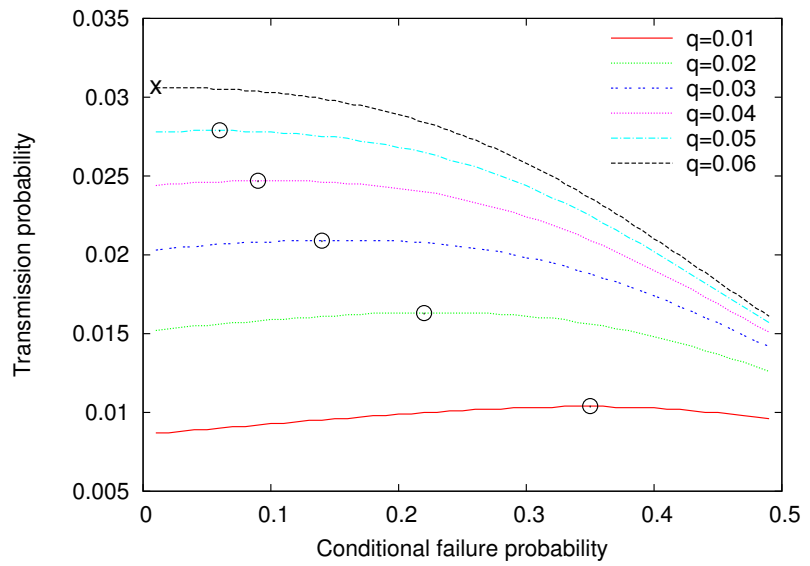

Figure 5. Relationship between $\tau$ and $p$ for different arrival rates $q$. The circles indicate the maximum values of $\tau$.

In Fig. 5 we plot the relationship between $\tau$ and $p$ as given by (11) for different $q$ values. We observe that, for non-saturated scenarios there exists a non-monotonic relationship between the transmission and collision probabilities, meaning an increase in $p$ can increase or decrease $\tau$. We also mark in the figure the turning point for each curve. To the left of this point increases in the failure probability result in increased transmission rate, and to the right increased failure probability results in decreased transmission rate. As $q$ increases, we see this point moves towards $p=0$. Following this observation, we could compute the $q$ value where the transmission rate becomes monotonic.

We conclude that, in the presence of capture effect, the throughput increase arises from a tradeoff between the transmission and success probabilities, depending on the stations' offered load, and cannot be understood in terms of only one of the aforementioned quantities alone.

\section{POWER-HOPPING MAC}

We have shown that in some circumstances the capture effect can boost the performance of all stations, as the increased success rate and the reduced number of retransmission experienced by nodes near the AP yield an increase in the air time available for all contenders. In this section, we take our finding one step further and we conjecture that, in dense deployments where nodes have similar channel conditions to the AP, by periodically setting the transmission power of stations to dissimilar levels, it would be possible to enhance the throughput performance of all stations. Therefore, we design a power-hopping MAC (PH-MAC) that exploits the capture effect to mitigate collisions, thereby outperforming the basic 802.11 DCF mechanism. We first quantify numerically the benefits of employing the proposed scheme and then present a prototype implementation. We conduct experiments with a real 802.11 deployment and demonstrate that PH-MAC improves throughput significantly without requiring changes to the existing hardware, 
but only some minor modification to the available opensource device drivers.

\section{A. PH-MAC Design}

Consider a situation where a station has determined that a power level $P$ is sufficient to transmit reliably to the AP at the selected PHY rate. Further, suppose that $P$ is less than the maximum transmission power, $P_{\max }$, supported by the hardware and the margin between $P$ and $P_{\max }$ is large enough to cause capture when two stations transmit simultaneously at these levels. In such circumstances, a station can use values from a range of powers levels and its transmission will still be successfully decoded at the AP when there is no collision, while allowing for stations transmitting at $P_{\max }$ to capture with different probabilities.

Following this observation, the objective of PH-MAC is to choose transmission powers so that a significant number of collisions result in capture. To this end, for each transmission attempt, PH-MAC randomly chooses between employing $P$ and $P_{\max }$ power levels. Specifically, we select the higher power $P_{\max }$ with probability $p_{h}$ and the lower power with probability $p_{l}=1-p_{h}$.

To understand how to properly configure these probabilities, let us first analyse the expected behaviour of our proposal. Consider a single cell 802.11 network with $n$ stations following the basic DCF rules. As described above, at each transmission attempt a station employs one of two power levels, randomly and independently chosen: high power with probability $p_{h}$ and low power with probability $p_{l}$. We assume transmissions with high power can capture the channel and thus be successful when encountering any transmissions with low signal strength. Conversely, transmissions at low power fail whenever they meet any other simultaneous transmission.

Initially, consider a symmetric network, where all stations have the same transmission rate $\tau$. Then, if all frames were transmitted with the same power level, the conditional collision probability $p_{c}$ could be written as

$$
p_{c}=1-(1-\tau)^{n-1}=\sum_{i=1}^{n-1}\left(\begin{array}{c}
n-1 \\
i
\end{array}\right) \tau^{i}(1-\tau)^{n-i-1} .
$$

However, since we employ the power hopping scheme, some of the simultaneous transmissions will be successfully decoded because of capture, while others will still fail. Thus, the failure probability $p$ is given by

$p=\sum_{i=1}^{n-1}\left(\begin{array}{c}n-1 \\ i\end{array}\right) \tau^{i}(1-\tau)^{n-i-1}\left(p_{h}\left(1-\left(1-p_{h}\right)^{i}\right)+\left(1-p_{h}\right)\right)$.

Using (11), we can solve the above and predict the throughput performance of the protocol for different values of $p_{h}$ if we apply (12). We employ this model to find the optimal $p_{h}$ value that maximises performance under powerhopping. For this purpose, we plot the throughput of a

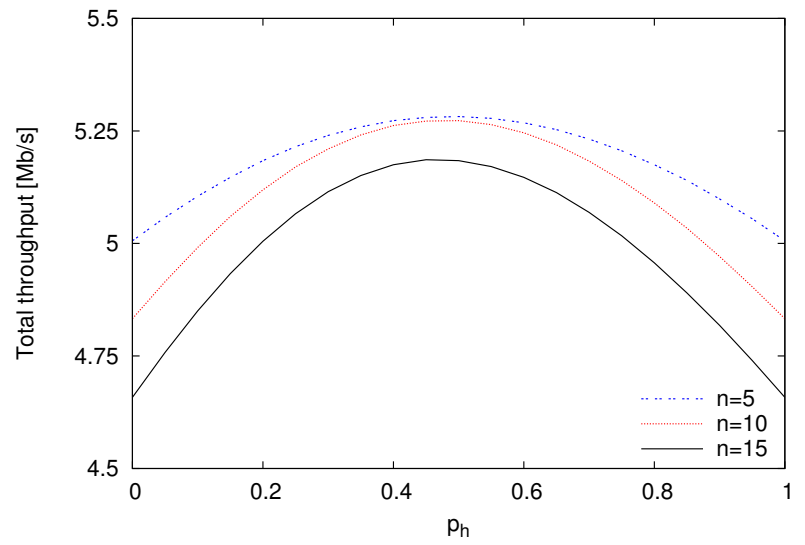

Figure 6. Throughput performance with power-hopping using different $p_{h}$ values, under saturation conditions.

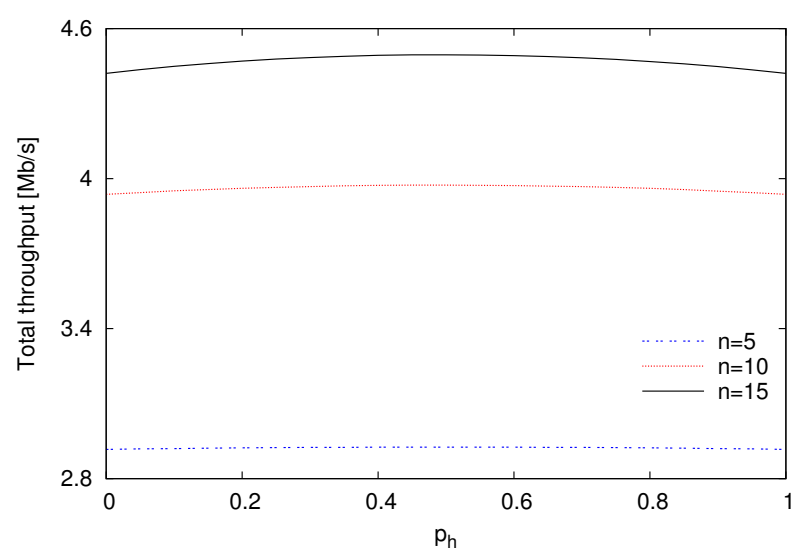

Figure 7. Throughput performance with power-hopping using different $p_{h}$ values, under non-saturation conditions.

saturated and non-saturated network in Figs. 6 and 7, respectively, for the possible range of $p_{h}$ values. Clearly, in both cases, choosing $p_{h}=0.5$ provides noticeable performance benefits as compared to the capture free scenario $\left(p_{h}=0\right.$ and $\left.p_{h}=1\right)$.

To understand the observed behaviour, remember that in practice $\tau$ takes small values (i.e. $\tau \ll 1$ ), therefore the term corresponding to $i=1$ dominates the expression of the conditional failure probability given by (16). Thus we can approximate $p$ by

$p \approx p_{c}\left(p_{h}^{2}+\left(1-p_{h}\right)\right)=\left(1-(1-\tau)^{n-1}\right)\left(p_{h}^{2}+\left(1-p_{h}\right)\right)$

More generally, we reason that, in a case where failures are dominated by collisions of two stations, the failure probability will be scaled by $p_{h}^{2}+\left(1-p_{h}\right)$ relative to the collision probability (of the capture free case). Since $p_{h}^{2}+\left(1-p_{h}\right)$ has a minimum at $p_{h}=0.5$, we can minimise the probability of failure by randomly choosing between high and low power levels with equal probabilities. 


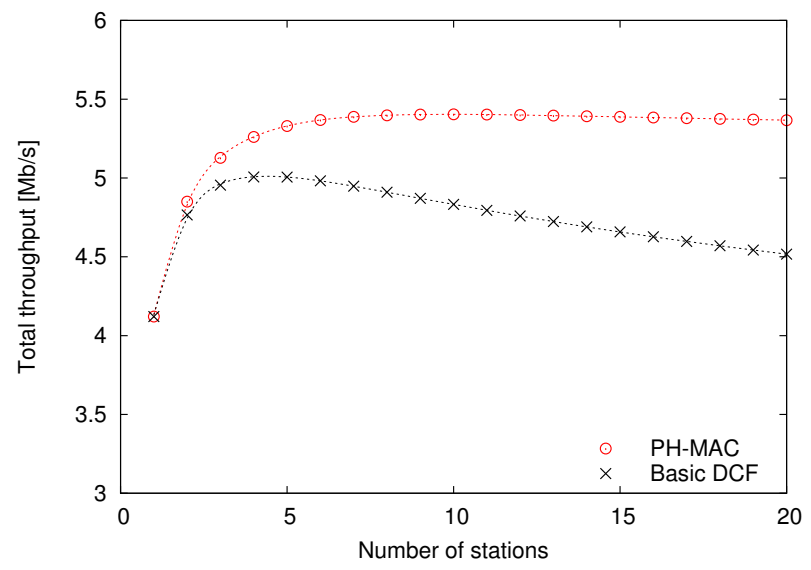

Figure 8. Throughput performance of PH-MAC compared with basic DCF, varying the network size, under saturation conditions.

\section{B. Numerical Results}

To estimate the benefits of using the proposed scheme, we first investigate numerically the throughput performance of PH-MAC as compared to the standard 802.11 MAC, varying the network conditions in terms of numbers of active stations and considering different offered loads.

We start by examining a network with saturated stations, sending again 500 byte packets and employing the $802.11 \mathrm{~b}$ parameters, and evaluate the throughput attained by $\mathrm{PH}-$ MAC and basic DCF as the number of active stations increases. As shown in Fig. 8, by randomly choosing between high and low power levels on each transmission, PH-MAC outperforms DCF, the throughput gain increasing with $n$. In particular, observe that PH-MAC alleviates much of the loss due to collisions in networks with more than five stations.

To understand the impact of the offered load on the performance of the proposed enhancement, we analyse a scenario in which 10 nodes are present in the WLAN and we consider different packet arrival rates. The results are illustrated in Fig. 9. We observe that, as the offered load increases, PH-MAC provides increasing throughput gains over the basic DCF mechanism. Note that, as we model stations with small buffers, the behaviour depicted in Fig. 9 corresponds to a system that has not reached saturation (i.e. $q<1$ ), hence the plotted throughput has not yet reached the value for $n=10$ stations shown in Fig. 8.

Following these results, we conclude that PH-MAC successfully exploits the capture effect to improve the performance of dense networks. These findings motivate a practical assessment of PH-MAC in real environments. To this end, we next present a prototype implementation, which we experimentally evaluate in a small-scale 802.11 testbed.

\section{Prototype Implementation}

We argue that the power-hopping enhancement we introduced can be effectively implemented with existing hardware, only with relatively basic driver modifications. To

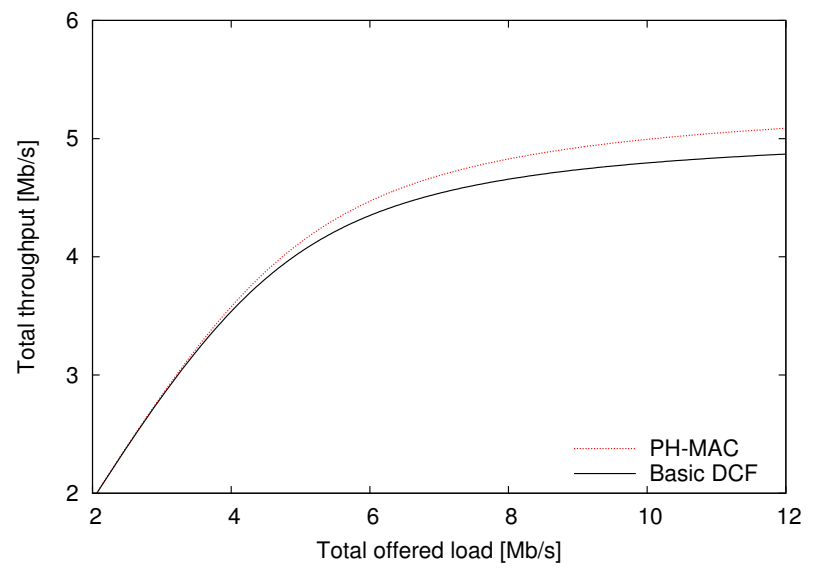

Figure 9. Throughput performance of PH-MAC compared with basic DCF, varying the offered load.

support our claim, in what follows we describe a prototype implementation we developed using the popular open-source MadWifi v0.9.4 driver. $^{3}$ Note that, due to the inherent limitations of the driver, which we discuss next, our prototype is an approximation of the proposed PH-MAC scheme. However, as our experimental results will show, the implemented prototype can significantly outperform the standard DCF in real deployments.

The key advantage of using MadWifi drivers with Atheros-based wireless cards over other practical 802.11 solutions is that they support Transmit Power Control (TPC) functionality, i.e. they allow setting the transmission power level with a per-packet granularity, as already reported in [16], [17]. In the PH-MAC approach, so far we have assumed that the power can be changed with each transmission attempt. However, this would require a fine control of the hardware, namely accessing the retransmission handling routines, that are generally embedded in the hardware abstraction layer (HAL) or firmware. Unfortunately, the source code implementing this low level functionality is not publicly available for the existing devices. Consequently, our prototype only tunes the transmission power on a per-packet instead of per-transmission attempt basis. Nonetheless, we expect only minor deviations from the predicted performance and we will demonstrate that, indeed, our implementation achieves noteworthy throughput gains despite this approximation.

To implement PH-MAC we modified the part of the driver's source code handling the transmission operations for the Atheros wireless LAN controller (if_ath). Precisely, when a new packet arrives at the MAC queue, an ath_tx_start routine is invoked to handle the transmission. After performing the encapsulation operations, computing the transmission duration and selecting the antenna to be used for transmission, the routine prepares a transmission

\footnotetext{
${ }^{3}$ http: //madwifi-project.org
} 


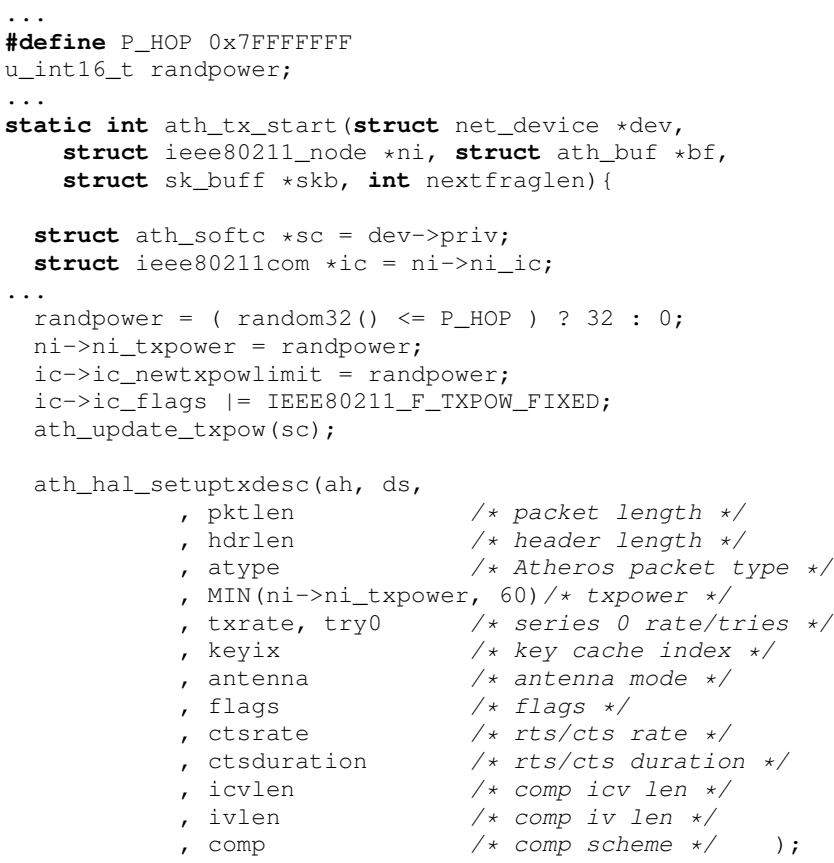

Figure 10. Driver modifications introduced by PH-MAC.

descriptor that is used to pass the packet to the hardware, along with a set of PHY layer parameters, among which the transmission power. To achieve the desired power-hopping functionality, before the transmission descriptor is provided to the hardware through the ath_hal_setuptxdesc call, we update the transmission power parameter retained in the node information structure ( $\mathrm{ni}$ ), that is passed at the call, and invoke the ath_update_txpow routine, that sets the TX power of the device to the desired level.

As already discussed, we randomly choose between a low and a high level with probability $p_{h}=p_{l}=0.5$. To this end, when enqueuing a packet, we generate a random unsigned 32-bit number using the random 32 routine, which we compare to the the value that splits in half the possible range, i.e. $0 \times 7 \mathrm{FFFFFFF}$, to select the transmit power. ${ }^{4}$ The power level passed for update has to be expressed as $0.5 \mathrm{dBm}$ increments, e.g. for setting the power to $P_{\max }=16 \mathrm{dBm}$ a value of 32 is expected. ${ }^{5}$ Note that MAC operation as specified by the standard is kept unmodified. In Fig. 10, we provide code snippets that implement the driver modifications required by PH-MAC. The source code of our implementation is publicly available online. ${ }^{6}$ In what follows we validate PH-MAC's operation in an 802.11 testbed.

\footnotetext{
${ }^{4}$ Although a predefined set of power levels can be configured, in our examples we select between the minimum and maximum admisible levels to obtain a high capture proability upon simultaneous transmissions.

${ }^{5}$ The maximum power level that can be set on a device varies with manufacturers and is subject to regulatory constraints.

${ }^{6}$ http: / / www.hamilton. ie/ppatras/\#code
}

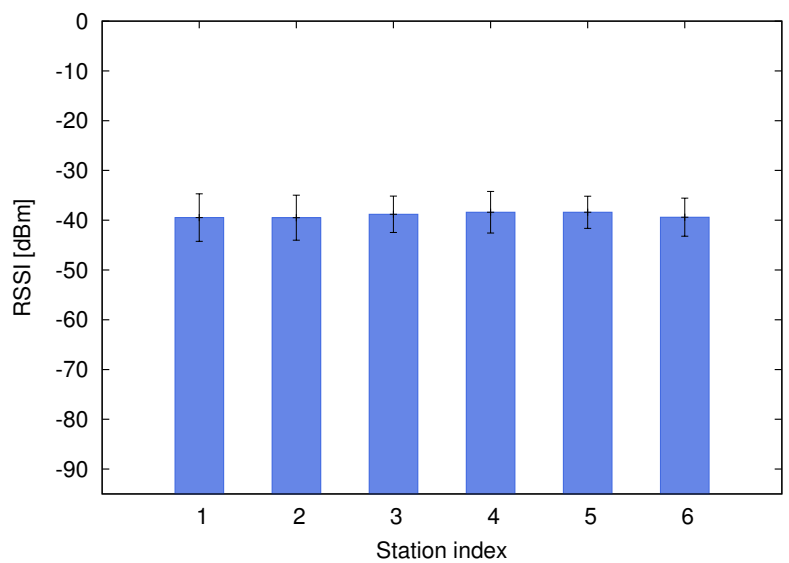

Figure 11. RSSI footprint of the testbed with nodes transmitting at $16 \mathrm{dBm}$.

\section{Experimental Evaluation}

To evaluate the potential performance benefits of $\mathrm{PH}$ MAC in a real environment we conduct experiments in a small testbed that we deployed in an office environment at the Hamilton Institute in Ireland. The testbed consists of 7 nodes: one PC acting as access point and 6 Soekris net4801 embedded PCs serving as clients. All nodes are equipped with Wistron CM9 Atheros miniPCI $802.11 \mathrm{~b} / \mathrm{g}$ cards, $5 \mathrm{dBi}$ omnidirectional antennas, and run the Ubuntu 8.04 LTS Linux distribution with kernel 2.6.24. The WLAN is operating on channel $6(2.437 \mathrm{GHz})$ where no other networks have been detected and thus we conclude it is an interference free environment. RTS/CTS, rate adaptation, turbo, fast frame, bursting and unscheduled automatic power save functionalities are disabled in all experiments, while the antenna diversity scheme is not employed for transmission/reception. All nodes are within LOS from the AP, thus we expect negligible multipath propagation effects.

Clients are placed at comparable distances from the AP to guarantee frames are received with similar signal strength, when stations are transmitting at the same power level. To confirm this, we periodically send ICMP traffic between each station and the AP, and record the received signal strength indicators (RSSI) for the received frames as reported in the radiotap header of the packets sniffed with tcpdump. In Fig. 11 we plot the average and standard deviation of the RSSI for each node sending at maximum TX power. We observe that, indeed, all nodes experience almost identical link qualities when sending to the AP.

Next, we set the data rate of each station at $11 \mathrm{Mb} / \mathrm{s}$, as all nodes were able to individually send frames to the AP without losses when employing this rate, regardless of the TX power employed, and use the iperf tool to generate saturated UDP traffic from each node towards the AP, varying the transmission power level within the possible range. As the packet error rate remains at $0 \%$ in all cases, we conclude that sending at low power levels (as when employing power-hopping) will not incur losses 


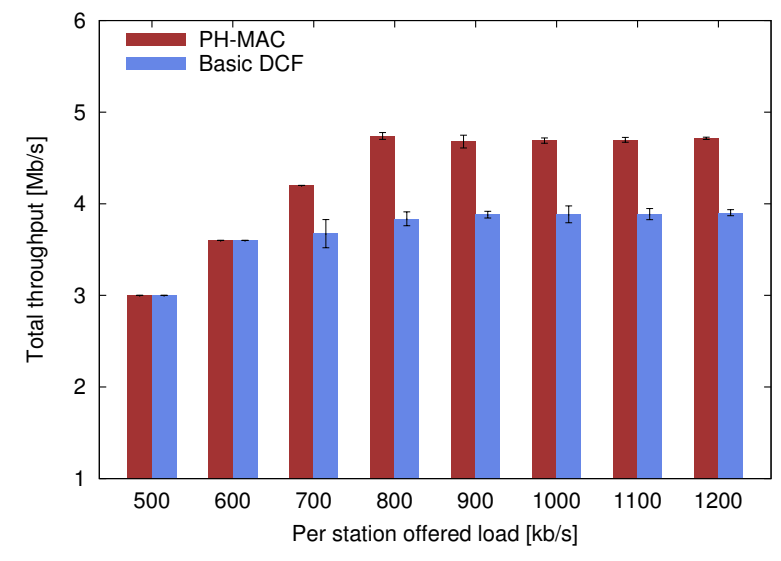

Figure 12. Experimental evaluation of PH-MAC.

due to fading. Finally, we set the TX power for half of the nodes at the minimum level $(0 \mathrm{dBm})$ and respectively at the maximum level $(16 \mathrm{dBm})$ for the other half, and configure all stations with a fixed $C W$, thereby fixing their transmission attempt rate to $\tau=2 /(C W+1)$. With this setting, all nodes simultaneously transmit saturated UDP flows to the AP. By knowing their transmission probabilities, estimating the failure probability from the traces recorded at the AP and applying (2), we conclude that a station sending at maximum power will capture the channel over stations transmitting concurrently at the minimum level with $\approx 0.8$ probability.

Following these preliminary measurements, we proceed with comparing the performance of PH-MAC and basic DCF for different traffic loads. Given the reduce size of our deployment we configure nodes with $C W_{\min }=7$ and $C W_{\max }=15$, to increase the level of contention in the WLAN when both basic DCF and PH-MAC are in operation. ${ }^{7}$ First, we enable PH-MAC at the stations to alternate between the minimum and maximum power levels for transmission, and again instruct clients to send UDP packets with 500 byte payload to the AP, this time varying the offered load and recording the total throughput. Further, we conduct the same set of tests with the network running the basic DCF mechanism and measure similarly the achieved performance. Each experiment runs for 3 minutes and is repeated 10 times to obtain average values of the throughput with good statistical significance.

The results are shown in Fig. 12, where we plot average and $95 \%$ confidence intervals of the throughput obtained with the two approaches. As observed in the figure, PHMAC significantly outperforms the basic DCF mechanism as stations generate more traffic, showing notable benefits as the network approaches saturation $(\lambda \geq 800 \mathrm{~kb} / \mathrm{s})$. In particular, by changing between low and high transmission power levels, PH-MAC achieves throughput gains of up to $25 \%$ over the standard default scheme.

\footnotetext{
${ }^{7}$ Note that these are realistic MAC settings, as they are employed for e.g. the video queue of the $802.11 \mathrm{a} / \mathrm{g}$ modes.
}

\section{Conclusions}

In this paper, we have studied the capture effect in 802.11 WLANs, with a view to understanding how it can enhance performance of all stations in the network. We have shown that, under particular circumstances, the capture effect can improve the throughput of both nodes delivering frames with high and low power, and provided insights into how these circumstances arise. Based on these findings, we designed PH-MAC, a power-hopping scheme that deliberately causes capture in a way that benefits all stations. We demonstrated PH-MAC's gains of up to $25 \%$ using both numerical simulations and experiments in a real deployment with a prototype implementation based on commodity hardware.

\section{REFERENCES}

[1] G. Bianchi, "Performance analysis of the IEEE 802.11 distributed coordination function," IEEE Journal on Selected Areas in Coтmunications (JSAC), vol. 18, no. 3, pp. 535-547, Mar 2000.

[2] H. Wu, Y. Peng, K. Long, S. Cheng, and J. Ma, "Performance of reliable transport protocol over IEEE 802.11 wireless LAN: analysis and enhancement," in Proc. IEEE INFOCOM, 2002, pp. 599-607.

[3] A. Kumar, E. Altman, D. Miorandi, and G. Munish, "New insights from a fixed-point analysis of single cell IEEE 802.11 WLANs," IEEE/ACM Trans. Netw., vol. 15, pp. 588-601, June 2007.

[4] D. Malone, K. Duffy, and D. Leith, "Modeling the 802.11 distributed coordination function in nonsaturated heterogeneous conditions," IEEE/ACM Trans. Netw., vol. 15, no. 1, pp. 159-172, Feb. 2007.

[5] C. Lau and C. Leung, "Capture models for mobile packet radio networks," in Proc. IEEE ICC, vol. 30, Apr. 1990, pp. 1226-1230.

[6] M. Zorzi and R. Rao, "Capture and retransmission control in mobile radio," IEEE Journal on Selected Areas in Communications (JSAC), vol. 12, no. 8, pp. 1289-1298, Oct. 1994.

[7] X. Ge, D. Yan, and Y. Zhu, "Throughput model of IEEE 802.11 networks with capture effect," in Proc. IEEE WiCOM, 2006, pp. 1-4.

[8] Z. Hadzi-Velkov and B. Spasenovski, "Capture effect in IEEE 802.11 basic service area under influence of Rayleigh fading and near/far effect," in Proc. IEEE PIMRC, vol. 1, 2002, pp. 172-176.

[9] — , "On the capacity of IEEE 802.11 DCF with capture in multipath-faded channels," International Journal of Wireless Information Networks, vol. 9, pp. 191-199, 2002.

[10] G. Sutton, R. Liu, X. Yang, and I. Collings, "Modelling capture effect for 802.11 DCF under Rayleigh fading," in Proc. IEEE ICCC, 2010.

[11] A. Kochut, A. Vasan, A. Vasan, A. U. Shankar, and A. Agrawala, "Sniffing out the correct physical layer capture model in $802.11 \mathrm{~b}$," 2004, pp. 252-261.

[12] J. Lee, W. Kim, S.-J. Lee, D. Jo, J. Ryu, T. Kwon, and Y. Choi, "An experimental study on the capture effect in 802.11a networks," in Proc. ACM WinTECH, Montreal, Quebec, Canada, 2007, pp. 19-26.

[13] N. Santhapuri, R. R. Choudhury, J. Manweiler, S. Nelakuduti, S. Sen, and K. Munagala, "Message in Message MIM: A case for reordering transmissions in wireless networks," in Proc. HotNets, 2008.

[14] IEEE 802.11 WG, Wireless LAN Medium Access Control (MAC) and Physical Layer (PHY) specifications. IEEE Std 802.11, 2007.

[15] K. Duffy, "Mean field Markov models of wireless local area networks," Markov Processes and Related Fields, vol. 16, 2010.

[16] K. Kowalik, M. Bykowski, B. Keegan, and M. Davis, "Practical issues of power control in IEEE 802.11 wireless devices," in Proc. IEEE ICT, june 2008, pp. 1-5.

[17] V. Shrivastava, D. Agrawal, A. Mishra, S. Banerjee, and T. Nadeem, "Understanding the limitations of transmit power control for indoor WLANS," in Proc. ACM IMC, San Diego, USA, 2007. 